\title{
ОЦЕНКА ЭНЕРГИИ ВОЗБУЖДЕННЫХ СОСТОЯНИЙ МЕТОДОМ, ПОДОБНЫМ ИСПОЛЬЗОВАНИЮ СООТНОШЕНИЯ НЕОПРЕДЕЛЕННОСТИ
}

\author{
(Представил П. Кард)
}

Для оценки энергии основного состояния часто используется соотношение неопределенности. Здесь мы покажем, что аналогичная техника применима также для оценки энергии возбужденных состояний.

Как известно, в обычном методе (см., напр., $\left.\left[{ }^{1}\right]\right)$ исходят из соотношения неопределенности в приближенной форме

$$
\xi p \simeq \hbar
$$

где $\zeta$ и $p-$ абсолютные величины координаты и импульса. Далее учитывается, что на основе ограничений, налагаемых соотношением неопределенности, можно еще с некоторой точностью использовать классическое выражение полной энергии

$$
E=p^{2} / 2 m+V(\zeta),
$$

где $m$ - масса частицы, $V(\zeta)$ - ее потенциальная энергия*. Затем, пользуясь формулой (1), выражают, например, величину $p$ через $\zeta$ и минимизируют выражение (2) по аргументу ל. Полученное минимальное значение толкуется как оценка энергии основного состояния. Точнее говоря, приводимые нами ниже соображения позволяют утверждать, что именно таким путем можно получить оценку энергии основного состояния.

На основе идеи, полученной из $\left[{ }^{2}\right]$, мы обобщаем формулу (1) следующим образом:

$$
\zeta p \simeq n \hbar,
$$

где $n$ - квантовое число, принимающее положительные целочисленные значения. Основой написания формулы (3) служит квазиклассическое приближение. В более наглядном толковании формула (3) выражает положение, согласно которому вдоль траектории частицы укладывается целое число длин волн де Бройля.

Выражая из формулы (3) p через ร, получаем для энергии приближенно

$$
E(\zeta)=n^{2} \hbar^{2} / 2 m \zeta^{2}+V(\zeta)
$$

Стационарные состояния как наиболее стабильные соответствуют минимуму энергии $E(\zeta)$ при заданном $n$, необходимым условием которого является $d E / d \zeta=0$, т. е.

$$
\hbar^{2} n^{2} / m \zeta_{n}^{3}=(d V / d \zeta)_{n}
$$

причем индекс $n$ у производной указывает на ее значение при $\zeta=\zeta_{n}$.

Достаточное условие минимума есть $d^{2} E / d \zeta^{2}>0$, т. е.

* В дальнейшем функция $V(\zeta)$ кратко называется потенциалом. 


$$
\left(d^{2} V / d \zeta^{2}\right)_{n}+3 \hbar^{2} n^{2} / m \zeta_{n}^{4}>0 .
$$

С учетом формулы (5) это условие можно переписать в виде

$$
\left(d^{2} V / d \zeta^{2}\right)_{n}+\left(3 / \zeta_{n}\right)(d V / d \zeta)_{n}>0 .
$$

Решение уравнения (5), удовлетворяющее условию (6), дает значение $\zeta_{n}$, характеризующее линейный размер системы в стационарном состоянии с квантовым числом $n$. Энергия $E_{n}$ в этом состоянии равна

$$
E_{n}=n^{2} \hbar^{2} / 2 m \zeta_{n}^{2}+V\left(\zeta_{n}\right) .
$$

Эту формулу полезно для практического применения переписать с учетом формулы (5) в форме

$$
E_{n}=V\left(\zeta_{n}\right)+\left(\zeta_{n} / 2\right)\left(d V / d \zeta_{n} .\right.
$$

Дифференцируя формулу (8) по квантовому числу $n$, имеем

$$
d E_{n} / d n=n \hbar^{2} / m \zeta_{n}^{2}+\left[(d V / d \zeta)_{n}-n^{2} \hbar^{2} / m \zeta_{n}^{3}\right]\left(d \zeta_{n} / d n\right) .
$$

В силу условия (5) выражение в квадратных скобках обращается в нуль и

$$
d E_{n} / d n=n \hbar^{2} / m \zeta_{n}^{2}>0 .
$$

Формула (11) показывает, что уровни энергии располагаются тем выше, чем больше квантовое число $n$. Таким образом, для основного состояния надо взять $n=1$. В этом случае из формулы (3) получается формула (1), которую раньше использовали для получения оценки энергии основного состояния. Для возбужденных состояний надо в формуле (3) взять $n>1$.

Чтобы определить характер зависимости $\zeta_{n}$ от $n$, возьмем производную по $n$ в выражении (5). Результат таков:

$$
d \zeta_{n} / d n=2 n \hbar^{2} / m \zeta_{n}^{3}\left[\left(d^{2} V / d \zeta^{2}\right)_{n}+\left(3 / \zeta_{n}\right)\left(d V / d \zeta_{n}\right] .\right.
$$

В силу формулы (7) $d \zeta_{n} / d n>0$, т. е. размер системы увеличивается с ростом квантового числа $n$.

Отметим, что с математической точки зрения предложенный нами метод имеет такую же структуру, как теория Бора в произвольном центрально-симметричном поле в случае круговых орбит (см., напр., $\left[{ }^{3}\right]$ ). Однако с методологической точки зрения достоинством предложенного метода является то, что он опирается на квантовую механику.

Перейдем к иллюстрации нашего метода. В качестве примера рассмотрим класс потенциалов, обладающих следующими свойствами:

a) кривая $d V / d \zeta$ пересекается только один раз с кривой $n^{2} \hbar^{2} / m \zeta^{3}$ при произвольном $n$;

б) выполняется условие

$$
d^{2} V / d \zeta^{2}+(3 / \zeta)(d V / d \zeta)>0
$$

при произвольном $\zeta$.

В рассматриваемый класс входят, очевидно, все потенциалы, для которых $d V / d \zeta$ является монотонно возрастающей функцией или постоянной, а также степенной потенциал

$$
V=k \zeta^{q}
$$

при $q>-2$, если $k q>0$. Это непосредственно вытекает из следующих простых формул: 


$$
\left.\begin{array}{l}
\begin{array}{l}
V / d \zeta=k q \zeta^{q-1} \\
d^{2} V / d \zeta^{2}+(3 / \zeta)(d V / d \zeta)=k q(q+2) \zeta^{q-2} .
\end{array}
\end{array}\right\}
$$

Действительно, из первой формулы видно, что для выполнения условия a) необходимо, чтобы $k q>0$ и $q \neq-2$, а из второй видно, что для выполнения условия б) надо иметь $q>-2$.

В частности, к виду (14) относятся случаи гармонического осциллятора и кулоновского потенциала

$$
V=-Z e^{2} / 4 \pi \varepsilon_{0} \zeta,
$$

написанного здесь в системе СИ для электрона водородоподобного атома.

Сравнение формул (1) и (3) позволяет для рассматриваемого класса потенциалов получить один замечательный результат. Для этого заметим, что в обсуждаемом здесь методе оценки энергии стационарных состояний постоянная $\hbar$ входит в вычисления только с помощью этих формул. Поскольку свойство потенциалов а) обеспечивает получение однозначното решения уравнения (5), а свойство б) обеспечивает выполнение условия (7) при произвольном $n$, то формулу для оценки энергии возбужденных состояний можно получить из формулы основного состояния путем простой подстановки

$$
\hbar \rightarrow n \hbar
$$

Ввиду произвольности $n$ этот результат означает, что для рассматриваемого класса потенциалов число уровней в данном приближении бесконечно велико.

В качестве примера можно взять кулоновский потенциал (16). Метод, основывающийся на формуле (1), дает оценку энергии основного состояния

$$
E_{1}=-Z^{2} m e^{4} / 32 \pi^{2} \varepsilon_{0}^{2} \hbar^{2} .
$$

Отсюда с помощью подстановки (17) получаем для возбужденных состояний формулу

$$
E_{n}=-Z^{2} m e^{4} / 32 \pi^{2} \varepsilon_{0}^{2} \hbar^{2} n^{2} .
$$

В заключение приведем еще конкретные вычисления для потенциала (14). Уравнение (5) решается элементарно:

$$
\zeta_{n}=\left(\hbar^{2} n^{2} / m k q\right)^{1 /(q+2)}
$$

Поскольку согласно свойству б) условие (7) выполнено, то $\zeta_{n}$ из формулы (20) можно сразу подставить в формулу (9) для получения оценки энергии стационарных состояний

$$
E_{n^{j}}=[\operatorname{sgn}(q)](1+q / 2)\left(\hbar^{2} n^{2}|k|^{2 / q} / m|q|\right)^{q /(q+2)} .
$$

Отсюда вытекает, что при $q<0 E_{n}<0$ и при $q>0 E_{n}>0$.

Отметим целесообразность применения именно формулы (9) вместо эквивалентной ей формулы (8). Последняя потребовала бы несколько более громоздких выкладок.

Если $q=-1$ и $k=-Z e^{2} / 4 \pi \varepsilon_{0}$ (кулоновский потенциал), то из (21) получается ранее известная формула (19). При $q>0$ наш результат (21) мало отличается от точного квазиклассического приближения (см., напр., $\left.\left[{ }^{4}\right]\right)$, где характер расположения уровней определяется множителем $(n+1 / 2)^{2 q /(q+2)}$. Если учесть, что формула (3), на которую опирается предложенный здесь метод оценки уровней, является грубым приближением, то полученный результат можно считать вполне приемлемым. 
1. Серова Ф. Г., Янкина А. А. Сборник задач по теоретической физике. Квантовая механика, статистическая физика. М., «Просвещение», 1979.

2. Шварц Н. А. В кн.: Второе зональное научно-методическое совещание заведующих кафедрами и ведущих лекторов по физике вузов Северо-Западной зоны. Л., ЛГУ, 1982, 17-19.

3. Lembra, J. В Kн.: Osakeste ja valguse maailmast. Tallinn, «Valgus», 1971, 33-40.

4. Sukhatme Uday, P. Amer, J. Phys., 41, № 8, 1015-1016 (1973).

Тартуский государственный университет

Поступила в редакцию 17/XII 1982

\section{J. LEMBRA}

\section{ERGASTATUD OLEKUTE ENERGIA HINNANG MEETODIL, MIS ON SARNANE MÃRAMATUSE RELATSIOONI KASUTAMISEGA}

Sageli lähtutakse põhioleku energia hindamisel määramatuse relatsioonist ligikaudsel kujul (1), kus $\zeta$ ja $p$ on vastavalt koordinaadi ja impulsi absoluutväärtused. Käesolevas töös on kvaasiklassikalise lähendi alusel lähtutud valemist (3), kus $n$ on kvantarv. Analoogiliste arvutustega saadakse juhul $n>1$ ergastatud oleku energia hinnang. Tulemus avaldub valemis (9), kus $\zeta_{n}$ on võrrandi (5) lahend, mis rahuldab tingimust (7). Meetodi illustreerimiseks on käsitletud astmepotentsiaali (14), mille energiatasemete hinnang on esitatud valemis (21).

\section{J. LEMBRA}

\section{THE ESTIMATE FOR ENERGY OF EXCITED STATES BY MEANS OF METHOD SIMILAR TO THE USE OF THE UNCERTAINTY PRINCIPLE}

Estimating of ground state energy is often started from the uncertainty principle in an approximate form (1), where $\zeta$ and $p$ are the absolute values of coordinate and momentum, respectively. On the basis of quasiclassical approximation the starting point is the formula (3) where $n$ is the quantum number. Carrying out analogical calculations, the estimate for the energy of excited states is received when $n>1$. The result is expressed by the formula $(9)$, where $\zeta_{n}$ is the solution of the equation (5), which obeys the condition (7). To illustrate the method, power potential (14) is considered, the estimate of energy levels of which is presented in the formula (21). 